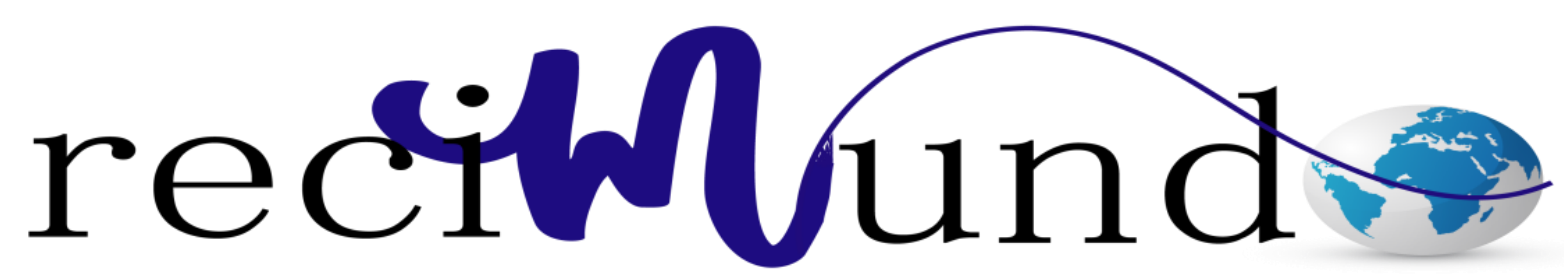

Revista Cientifica Mundo de la Investigación y el Conocimiento

\author{
Nakin Alberto Véliz Mero a; Marco Vinicio Peñaherrera Alcívar ${ }^{\text {b; }}$ Martha Saida \\ Quiroz Figueroa ${ }^{c}$; Hilda Monserrate Mendoza Sornoza ${ }^{d}$; Jaime Eduardo Jaramillo \\ Soto ${ }^{\mathrm{e}}$; Mónica Danny Tonguino Rodríguez ${ }^{\mathrm{f}}$
}

Prevención frente la presencia de anemia en el embarazo

Prevention against the presence of anemia in pregnancy

Revista Científica Mundo de la Investigación y el Conocimiento. Vol. 3 núm.1, enero, ISSN: 2588-073X, 2019, pp. 971-996

DOI: 10.26820/recimundo/3.(1).enero.2019.971-996

URL: http://www.recimundo.com/index.php/es/article/view/402

Editorial Saberes del Conocimiento

Recibido: 20/11/2018 Aceptado: 05/01/2019 Publicado: 31/01/2019

Correspondencia: nakin.veliz@uleam.edu.ec

a. Doctor en Medicina y Cirugía; Magister en Gerencia de Salud para el Desarrollo Local, Magister en Gestión y Desarrollo Social; Universidad Laica Eloy Alfaro de Manabí; nakin.veliz@uleam.edu.ec

b. Médico General; Maestrante en Seguridad y Salud Laboral; dr.marco.palcivar@hotmail.com

c. Licenciada en Enfermería; Magíster en Investigación Clínica y Epidemiología; Docente Universidad Estatal del Sur de Manabí; marthaquiroz85@hotmail.com Medico general; Hospital Básico Jipijapa; hildamendoza21@ hotmail.com

d. Médico General; Hospital Dr. Gustavo Domínguez Zambrano; jaramillo666@ hotmail.com

e. Licenciada en Enfermería; moni tonguino@outlook.com 


\section{La complejidad en la relación médico paciente: Una mirada actual}

Vol. 3, núm. 1., (2019)

Nakin Alberto Véliz Mero; Marco Vinicio Peñaherrera Alcívar; Martha Saida Quiroz Figueroa; Hilda Monserrate Mendoza Sornoza; Jaime Eduardo Jaramillo Soto; Mónica Danny Tonguino Rodríguez

\section{RESUMEN}

El embarazo es un estado extraordinario donde las mujeres en edad reproductiva experimentan cambios fisiológicos que representan riesgos y complicaciones como es la presencia de anemia. Se considera anemia en el embarazo cuando la concentración de hemoglobina $(\mathrm{Hb})$ es menor de $11.0 \mathrm{~g} / \mathrm{dL}$ durante el primer y tercer trimestre, o menor de $10.5 \mathrm{~g} / \mathrm{dL}$ durante el segundo trimestre. La ferritina sérica durante la gestación disminuye incluso en mujeres que ingieren suplementos diarios de hierro, en general se recomienda prevenir mediante modificaciones de la dieta, fortificación de los alimentos y suplementación con hierro nutricional. Ninguna de estas estrategias es excluyente. La forma ideal de prevenir la carencia de hierro es mediante una dieta adecuada, lo que no siempre es posible de lograr por limitaciones económicas o hábitos muy arraigados. Es necesario promover el consumo de alimentos biodisponibles en hierro (como las carnes) mediante la educación nutricional y difundir información sobre las consecuencias en la salud que conlleva la anemia y la deficiencia de hierro. Adicionalmente promover y apoyar la lactancia materna exclusiva para mantener las reservas corporales de hierro después del parto, se recomiendan los suplementos de dosis bajas de hierro oral durante la segunda mitad del embarazo en las mujeres sin riesgo de ferropenia. En pacientes con anemia previa se debe iniciar al principio de la gestación.

Palabras claves: Edad Reproductiva; Anemia; Dieta Adecuada; Deficiencia de Hierro, Embarazo en las Mujeres. 


\section{La complejidad en la relación médico paciente: Una mirada actual}

Vol. 3, núm. 1., (2019)

Nakin Alberto Véliz Mero; Marco Vinicio Peñaherrera Alcívar; Martha Saida Quiroz Figueroa;

Hilda Monserrate Mendoza Sornoza; Jaime Eduardo Jaramillo Soto; Mónica Danny Tonguino

Rodríguez

\section{ABSTRACT}

Pregnancy is an extraordinary state where women of reproductive age experience physiological changes that represent risks and complications such as the presence of anemia. It is considered anemia in pregnancy when the concentration of hemoglobin $(\mathrm{Hb})$ is less than $11.0 \mathrm{~g} / \mathrm{dL}$ during the first and third trimesters, or less than $10.5 \mathrm{~g} / \mathrm{dL}$ during the second trimester. Serum ferritin during pregnancy decreases even in women who ingest daily iron supplements, in general it is recommended to prevent changes in diet, fortification of food and supplementation with nutritional iron. None of these strategies is exclusive. The ideal way to prevent iron deficiency is through an adequate diet, which is not always possible to achieve due to economic limitations or deep-rooted habits. It is necessary to promote the consumption of bioavailable iron foods (such as meats) through nutritional education and disseminate information on the health consequences of anemia and iron deficiency. In addition to promoting and supporting exclusive breastfeeding to maintain body iron stores after delivery, low-dose oral iron supplements are recommended during the second half of pregnancy in women without risk of iron deficiency. In patients with previous anemia should be started at the beginning of pregnancy.

Keywords: Reproductive Age; Anemia; Adequate Diet; Iron Deficiency; Pregnancy in Women. 


\section{La complejidad en la relación médico paciente: Una mirada actual}

Vol. 3, núm. 1., (2019)

Nakin Alberto Véliz Mero; Marco Vinicio Peñaherrera Alcívar; Martha Saida Quiroz Figueroa; Hilda Monserrate Mendoza Sornoza; Jaime Eduardo Jaramillo Soto; Mónica Danny Tonguino Rodríguez

\section{Introducción.}

Durante la gestación la poca disponibilidad de hierro almacenado puede desarrollar anemia, es decir las mujeres que no tienen la cantidad apropiada de hierro frente al embarazo genera complicaciones en la madre y el bebe, puesto que el feto necesita de los glóbulos rojos de la madre para su crecimiento y desarrollo, por lo tanto es necesario que se realice una adecuada suplementación, como es el caso de tratamientos prolongados con la administración de hierro en la prevención de anemia.

El embarazo induce cambios fisiológicos. Entre éstos, uno de los más significativos es el aumento en la volemia. Esta variación normal produce anemia fisiológica del embarazo. La concentración de hemoglobina es más baja durante el segundo y tercer trimestre. Durante el primer trimestre y muy cerca del final del embarazo, la concentración de hemoglobina de la mayoría de las mujeres sanas y con adecuadas reservas de hierro es de 11,5 g/L. La anemia durante el embarazo es generalmente aceptada como resultado de deficiencias nutricionales. Constituye un problema mayor de salud pública. Es la más frecuente de las enfermedades que pueden coincidir con el embarazo o ser producidas por este, ya que las necesidades para el desarrollo del feto y la placenta aumenta el consumo de hierro elemental. (ACOG; 2008).

La anemia es uno de los problemas nutricionales de mayor magnitud en el mundo, fundamentalmente en los países emergentes y se presenta con frecuencia en la mujer embarazadas lo que termina afectando al recién nacido. Varios estudios a nivel mundial han demostrado una prevalencia elevada de anemia dentro de las 24 horas de vida. (López \& Alvarez; 2010) y (Hernández; 2006). 


\section{La complejidad en la relación médico paciente: Una mirada actual}

Vol. 3, núm. 1., (2019)

Nakin Alberto Véliz Mero; Marco Vinicio Peñaherrera Alcívar; Martha Saida Quiroz Figueroa;

Hilda Monserrate Mendoza Sornoza; Jaime Eduardo Jaramillo Soto; Mónica Danny Tonguino

Rodríguez

Los tipos de anemia más frecuentes son la ferropénica, megaloblástica y de células

falciformes. La anemia por deficiencia de hierro es la más común en países subdesarrollados y representan $75 \%$ de los casos aproximadamente. Esto se debe, en la mayoría de los casos, a malnutrición durante el embarazo y déficit en el diagnóstico prenatal de anemia. (Espitia \& Orozco; 2013).

La ferropenia y la anemia por deficiencia de hierro constituyen importantes problemas nutricionales que afectan la salud de millones de mujeres y niños principalmente en comunidades pobres de países dependientes. Durante la gestación, los bajos depósitos de hierro maternos, la poca disponibilidad de este mineral en la dieta y la inadecuada utilización de un suplemento de hierro y ácido fólico, contribuyen a la insuficiente producción de hemoglobina y finalmente a la presencia de anemia. (Siega et al.; 2006).

Es imposible con la dieta habitual llegar a cifras adecuadas de concentración de hierro durante el embarazo y por lo tanto es necesario que se realice una adecuada suplementación. (Tojo; 2001).

Las anemia por carencias de hierro en la dieta y sus posibles complicaciones en la madre y el bebe son fácilmente prevenibles con el uso la suplementación de sulfato ferroso. Se sabe que tratamientos prolongados como es el caso de la administración de hierro en la prevención de anemia, con sus efectos adversos sumada a la falta de percepción clínica de las pacientes de las anemias leves, son causas de poca adherencia al tratamiento. (Peralta, \& Carbajal; 2008) y (Silva et al.; 2005). 


\section{La complejidad en la relación médico paciente: Una mirada actual}

Vol. 3, núm. 1., (2019)

Nakin Alberto Véliz Mero; Marco Vinicio Peñaherrera Alcívar; Martha Saida Quiroz Figueroa; Hilda Monserrate Mendoza Sornoza; Jaime Eduardo Jaramillo Soto; Mónica Danny Tonguino Rodríguez

Es importante tener en cuenta que durante el embarazo suele presentarse una anemia por dilución o fisiológica, debido a cambios propios de la gestación (aumento del volumen plasmático, disminución del hematocrito, entre otros). Por este motivo, es necesaria la suplementación con hierro y ácido fólico durante el embarazo, con el propósito de prevenir cuadros de anemia gestacional y asegurar el bienestar del binomio materno-fetal. (Manjarrés et al.; 2012).

La nutrición es una ciencia de gran importancia para el profesional de la salud, pues permite estudiar cuáles sustancias nutritivas, así como la cantidad y calidad, deben ingerirse para mantener el crecimiento, la reproducción normal y un buen estado de salud; también posibilita la investigación de cada uno de los nutrientes que ayudan en las funciones fisiológicas, entre los que se encuentran: glúcidos, lípidos, proteínas, vitaminas y minerales. La inadecuada alimentación provoca múltiples enfermedades como consecuencias de deficiencias o excesos de algún nutrimento. Se ha señalado que el déficit de hierro es la causa más frecuente de anemia, en el mundo, y el trastorno orgánico más habitual en la práctica médica. Su mayor prevalencia se encuentra en los países subdesarrollados. (Yip, R.; 2003).

\section{Metodología.}

El presente artículo tiene una visión específica, Se ha cumplido con una revisión de literatura en diferentes bases de datos electrónicas académicas de la cual se hizo la revisión más próxima considerando palabras claves que reseñen el tema a estudiar. Para la realización de la actual investigación, se consideró información confiable sobre trabajos publicados en versados depósitos de investigación en los que se estudia las implicaciones de salud que puede enfrentar 


\section{La complejidad en la relación médico paciente: Una mirada actual}

Vol. 3, núm. 1., (2019)

Nakin Alberto Véliz Mero; Marco Vinicio Peñaherrera Alcívar; Martha Saida Quiroz Figueroa;

Hilda Monserrate Mendoza Sornoza; Jaime Eduardo Jaramillo Soto; Mónica Danny Tonguino

Rodríguez

una mujer en estado de gravidez al presentar anemia, se utilizaron informaciones bibliográficas,

se la obtuvo de libros electrónicos, informes, artículos científicos y revistas, descartando toda información disponible sin verificación de fuente.

\section{Resultados.}

La anemia en el embarazo

Anemia es el estado patológico o enfermedad sistémica, en el cual, debido a la insuficiencia de glóbulos rojos (masa de heritrocitos circulantes), el aporte de oxígeno adherido a la heteroproteina hemoglobina, alteran la función celular, predominantemente nerviosa y muscular. El feto depende de la sangre materna para el aporte suficiente de este compuesto orgánico no metal (oxígeno), por tanto la anemia puede ocasionar un crecimiento fetal deficiente, un nacimiento prematuro y un bebé con bajo peso al nacer. Para determinar que se trata de anemia, debe existir una reducción mayor al $10 \%$ en la concentración de hemoglobina en la sangre periférica por debajo de lo normal, de acuerdo a la edad, género y altura sobre el nivel del mar. (Anivarro; 2007).

La baja ganancia de peso en la gestante se asocia con retardo del crecimiento intrauterino, al respecto, en un estudio realizado por (Strauss y Dietz; 1999), se examinó dicha relación en 10.696 mujeres, para lo cual se determinó como baja ganancia en el primer trimestre un incremento inferior a $0,1 \mathrm{~kg} / \mathrm{semana}$; en el segundo y tercer trimestre un aumento menor de 0,3 $\mathrm{kg} / \mathrm{semana}$. No se encontró asociación entre la ganancia de peso materno en el primer trimestre con un incremento en el riesgo de retardo del crecimiento intrauterino (RCI), pero si se halló relación con la baja ganancia de peso en el segundo y tercer trimestre. De acuerdo con lo 


\section{La complejidad en la relación médico paciente: Una mirada actual}

Vol. 3, núm. 1., (2019)

Nakin Alberto Véliz Mero; Marco Vinicio Peñaherrera Alcívar; Martha Saida Quiroz Figueroa; Hilda Monserrate Mendoza Sornoza; Jaime Eduardo Jaramillo Soto; Mónica Danny Tonguino Rodríguez

anterior, la ganancia de peso en la segunda mitad de la gestación es crítica para el desarrollo del feto. Mujeres con IMC bajo están en riesgo de un nacimiento pretérmino, que se incrementa si ellas han tenido un embarazo previo con parto prematuro. (Restrepo, Parra; 2009).

La anemia se define como una disminución de la cantidad de hemoglobina circulante, asociada generalmente a una disminución en el número de glóbulos rojos, que se traduce finalmente en un deterioro en la capacidad de transporte de oxígeno. La existencia de esta condición obedece a múltiples causas, una de ellas es la carencia de algunos micronutrientes (vitaminas y minerales) que deben ser aportados por la dieta, la falta de estos elementos necesarios en la formación y desarrollo de la serie roja condiciona un gran impacto sobre el desarrollo físico y mental del individuo. (Urdampilleta et al.; 2010).

Una anemia a la que no se modifique, puede causar trastornos en el aprendizaje, aumentar el riesgo de infecciones, reducir la capacidad de trabajo y provocar la muerte de la mujer durante el embarazo o parto; esta también se asocia con trastornos del embarazo, bajo peso al nacer, prematuridad, afecciones en el recién nacido y mortalidad materna y perinatal. (Gay; 1998).

En la mujer adulta existen 2 factores que predisponen a desarrollar anemia ferropénica: la menorragia (pérdida excesiva de sangre durante la menstruación) y el embarazo. Alrededor de 10 $\%$ de las mujeres experimentan pérdidas importantes de sangre con las reglas (>80 mL/mes), lo que a menudo da lugar a este tipo de anemia. Los anticonceptivos intrauterinos aumentan la menorragia en 30 a $50 \%$. Es característico que las mujeres con menorragia no sean conscientes de tener pérdidas de sangre superiores a las normales, razón por la cual resulta aconsejable hacer 


\section{La complejidad en la relación médico paciente: Una mirada actual}

Vol. 3, núm. 1., (2019)

Nakin Alberto Véliz Mero; Marco Vinicio Peñaherrera Alcívar; Martha Saida Quiroz Figueroa;

Hilda Monserrate Mendoza Sornoza; Jaime Eduardo Jaramillo Soto; Mónica Danny Tonguino

Rodríguez

una detección sistemática de la anemia, como parte de los exámenes habituales de salud. (OMS; 2003) у (Gay; 1998).

La anemia severa en el embarazo se define como una hemoglobina $<70 \mathrm{~g} / \mathrm{L}$ ( $7 \mathrm{~g} / \mathrm{dl})$ y que requiera tratamiento médico y la anemia muy severa se define como hemoglobina <40 g/L (4 g/dl) y representa una emergencia médica debido al riesgo de insuficiencia cardíaca congestiva. (WHO; 2011).

Toda anemia en el embarazo debe ser evaluada para ser diferenciada de una anemia patológica dado que anemias severas con hemoglobina por debajo de $6 \mathrm{~g} / \mathrm{dL}$ se han asociado con un volumen reducido de líquido amniótico, vasodilatación cerebral fetal, patrones de frecuencia cardíaca anormales, prematuridad, aborto espontáneo, bajo peso al nacer y muerte fetal. (Kilpatrick, et al.; 2017) y (WHO; 2001).

Se considera anemia en el embarazo cuando la concentración de hemoglobina (Hb) es menor de $11.0 \mathrm{~g} / \mathrm{dL}$ durante el primer y tercer trimestre, o menor de $10.5 \mathrm{~g} / \mathrm{dL}$ durante el segundo trimestre. La ferritina sérica durante la gestación disminuye incluso en mujeres que ingieren suplementos diarios de hierro, lo que pone en duda su utilidad como parámetro de control pero, a pesar de ello, se acepta que una concentración de ferritina menor de $12 \mu \mathrm{g} / \mathrm{L}$ indica agotamiento de las reservas de hierro. (López et al.; 2010).

Se sabe que niveles de $\mathrm{Hb}$ menores de $9.5 \mathrm{~g} / \mathrm{dL}$ antes o durante el segundo trimestre, o inferiores a $11.0 \mathrm{~g} / \mathrm{dL}$ cerca del término se asocian con bajo peso al nacer, prematuridad y al aumento de la mortalidad perinatal, sobre todo cuando son inferiores a 8.5-9.5 g/dL. Además, el déficit de hierro perjudica el rendimiento cognitivo y el desarrollo físico de los recién nacidos. 


\section{La complejidad en la relación médico paciente: Una mirada actual}

Vol. 3, núm. 1., (2019)

Nakin Alberto Véliz Mero; Marco Vinicio Peñaherrera Alcívar; Martha Saida Quiroz Figueroa; Hilda Monserrate Mendoza Sornoza; Jaime Eduardo Jaramillo Soto; Mónica Danny Tonguino Rodríguez

Los niveles de $\mathrm{Hb}$ elevados por encima de $13.5 \mathrm{~g} / \mathrm{L}$ se han asociado a hemoconcentración, hiperviscosidad sanguínea, con disminución de la perfusión placentaria, preeclampsia, eclampsia y crecimiento intrauterino retardado (CIR). (López et al.; 2010).

La anemia ferropénica puede desarrollarse durante el embarazo debido al aumento de las necesidades de hierro destinado al volumen sanguíneo materno en expansión y al rápido crecimiento del feto y la placenta. Se hace difícil que la dieta suministre la cantidad de hierro requerida durante la segunda mitad del embarazo, por lo que crece el peligro de deficiencia de hierro, sobre todo cuando se acerca el final de la gestación. La cantidad media de hierro total de una mujer sana no embarazada es de unos $2,3 \mathrm{~g}$, de los que solo, aproximadamente, 0,3 corresponden a depósitos del metal. (Yip, R.; 2003).

La cantidad total de hierro necesario durante el embarazo es, aproximadamente de $1 \mathrm{~g}$, lo cual supera, en gran medida, la cantidad de la que disponen en sus depósitos la mayor parte de las mujeres. A medida que el embarazo progresa, la disminución de los depósitos de hierro aumenta la eficacia de la absorción del contenido en la dieta; no obstante, algunas mujeres sufrirán depleción de sus depósitos de hierro y se harán anémicas, puesto que el riesgo de anemia ferropénica es bastante elevado. Durante el embarazo son necesarios los suplementos preventivos del hierro como práctica de rutina. (Yip, R.; 2003).

Existen múltiples indicadores bioquímicos y hematológicos para evaluar el estado de hierro corporal materno, sin embargo la ferritina, la hemoglobina y algunos índices eritrocitarios, son los más utilizados para el diagnóstico de la deficiencia de hierro y la anemia, puesto que son 


\section{La complejidad en la relación médico paciente: Una mirada actual}

Vol. 3, núm. 1., (2019)

Nakin Alberto Véliz Mero; Marco Vinicio Peñaherrera Alcívar; Martha Saida Quiroz Figueroa;

Hilda Monserrate Mendoza Sornoza; Jaime Eduardo Jaramillo Soto; Mónica Danny Tonguino

Rodríguez

de bajo costo, fácil accesibilidad y cuentan con métodos estandarizados para su análisis. (Parra; 2007) y (Mei et al.; 2005).

Estudios prospectivos también han mostrado la asociación entre anemia en el embarazo y bajo peso al nacer, sin embargo, no se ha establecido la relación de causalidad, puesto que existen otros factores que pueden contribuir a la anemia y por tanto generar bajo peso neonatal como es el caso de la desnutrición materna grave con incapacidad para incrementar la masa eritrocitaria o lograr la adecuada expansión del volumen sanguíneo por deficiente síntesis de albúmina. (Restrepo \& Parra; 2009).

Anemia, o más correcto oligohemia, es un síntoma común a muchas enfermedades, consistente en la disminución absoluta del caudal hemoglobínico (oligocromenia) del organismo, a la que suele acompañar una disminución de la cifra de hematíes (oligocitemia). Las formas subclínicas (sin sintomatología anémica manifiesta) son frecuentes durante el embarazo. Se diagnostican en los hemogramas rutinarios. (Ramírez; 2009)

El déficit de ácido fólico también se ha asociado con niveles elevados de homocisteína en sangre. La homocisteína es un aminoácido no esencial que posee efectos teratógenos y éste podría ser el mecanismo de actuación en los DTN. Además, también podría ser responsable de algunas situaciones mediadas por una vasculopatía placentaria como el aborto espontáneo, el desprendimiento prematuro de placenta y la preeclampsia. El aborto de repetición podría estar provocado por dos mecanismos: por un lado, por el efecto embriotóxico directo de la homocisteína y por otro, por la lesión vascular en la decidua, con alteración en las vellosidades coriales. (López et al., 2010). 


\section{La complejidad en la relación médico paciente: Una mirada actual}

Vol. 3, núm. 1., (2019)

Nakin Alberto Véliz Mero; Marco Vinicio Peñaherrera Alcívar; Martha Saida Quiroz Figueroa; Hilda Monserrate Mendoza Sornoza; Jaime Eduardo Jaramillo Soto; Mónica Danny Tonguino Rodríguez

En cuanto a la preeclampsia, la hiperhomocisteinemia puede provocar lesiones endoteliales y cambios metabólicos que contribuyen a que se manifieste la enfermedad. El ácido fólico también tiene repercusión en la salud de la población general. Parece disminuir la incidencia de cáncer de colon y mama y en un estudio reciente se mostró que la suplementación de ácido fólico durante el embarazo reduce el riesgo de leucemia linfoblástica aguda infantil en un 60\%. (López et al.; 2010).

\section{Alimentación y nutrición}

La nutrición considera el conjunto de procesos por los cuales el cuerpo humano digiere, absorbe, transforma, utiliza y elimina sustancias nutritivas contenidas en los alimentos con el fin de obtener energía, construir y reparar las estructuras corporales, así como regular el metabolismo. Este es un proceso metabólico, involuntario y no educable, por el cual se procuran los alimentos necesarios para mantener la vida; los que se seleccionan, según las disponibilidades; preparan, acorde a los gustos y costumbres y, finalmente, se ingieren. Es, además, un proceso voluntario, educable y muy influenciable por factores sociales, económicos, culturales y otros. Los alimentos aportan las sustancias nutritivas o nutricionales que son: proteínas, grasas, carbohidratos, vitaminas y minerales, que contenidas en los alimentos van a aportar materiales plásticos, energéticos y factores de regulación indispensables para su funcionamiento. (Yip, R.; 2003).

Al analizar el perfil nutricional de los países, se tiene que considerar que las últimas mediciones disponibles reflejan prevalencias de desnutrición global equivalentes a entre 1,7 y 3,4 veces lo normal, siendo Ecuador el país con más alta prevalencia $(8,6 \%)$, seguido por Perú 


\section{La complejidad en la relación médico paciente: Una mirada actual}

Vol. 3, núm. 1., (2019)

Nakin Alberto Véliz Mero; Marco Vinicio Peñaherrera Alcívar; Martha Saida Quiroz Figueroa;

Hilda Monserrate Mendoza Sornoza; Jaime Eduardo Jaramillo Soto; Mónica Danny Tonguino

Rodríguez

(7,6\%), Bolivia $(7,5 \%)$ y Colombia $(7,0 \%)$. En tanto Venezuela y Paraguay presentan

prevalencias significativamente menores ( $4,6 \%$ y 4,2\%, respectivamente). (Abrams et al.; 2000).

La conveniencia del uso rutinario de suplementos de hierro durante el embarazo, independientemente de si la madre es anémica o no, ha sido debatida principalmente en Estados Unidos e Inglaterra y no es universalmente aceptada por los países industrializados. A pesar de ello, múltiples estudios concluyen que el suplemento de hierro mejora el estado nutricional de las gestantes, aumenta los niveles de hemoglobina, hematocrito, el volumen corpuscular medio, entre otros indicadores y esto a su vez ejerce influencia en la salud y el peso de los neonatos, tanto en los países desarrollados como en los dependientes. (Restrepo \& Parra; 2009).

En cuanto a las deficiencias nutricionales, es importante tener en cuenta que en la mayoría de las mujeres, los requerimientos de hierro en la segunda mitad del embarazo se aproximan a $27 \mathrm{mg} /$ día. Esta cantidad de hierro no están presentes en los depósitos intracelulares, razón por la que, sin suplemento de hierro, no se desarrolla el volumen de eritrocitos maternos necesarios, y la concentración de $\mathrm{Hb}$ y el Hto disminuye considerablemente a medida que aumenta el volumen plasmático. La ingesta inadecuada de hierro antes y durante el embarazo es la principal causa de anemia por deficiencia de hierro. Esta deficiencia de hierro se manifiesta con una disminución apreciable de la concentración de hemoglobina, generalmente durante el tercer trimestre. Por las razones anteriormente mencionadas, es fundamental la suplementación con hierro durante el embarazo para la prevención de cuadros anémicos. (Martínez et al.; 2018).

Según Roca Goderich, R., (2002). Dentro de las anemias nutricionales se distinguen:

a) Anemia ferropénica (producida por deficiencia de hierro). 


\section{La complejidad en la relación médico paciente: Una mirada actual}

Vol. 3, núm. 1., (2019)

Nakin Alberto Véliz Mero; Marco Vinicio Peñaherrera Alcívar; Martha Saida Quiroz Figueroa; Hilda Monserrate Mendoza Sornoza; Jaime Eduardo Jaramillo Soto; Mónica Danny Tonguino Rodríguez

b) Anemia megaloblástica (causada por la carencia de vitamina B12 y folatos).

Se debe orientar la alimentación con énfasis en los aspectos cualitativos y las combinaciones de alimentos que favorezcan la biodisponibilidad de los micronutrientes.

El hierro heme está presente en la carne, el pescado y las aves de corral, así como en los productos sanguíneos. El no heme es una fuente más importante y se encuentra, en diversa medida, en todos los alimentos de origen vegetal. Las modificaciones de la dieta incluyen aumento del consumo de alimentos ricos en sustancias que favorecen la asimilación del hierro no heme, disminución de la ingestión de inhibidores de la absorción y elevación del consumo del hierro heme. (Yip, R.; 2003)

El estado nutricional materno desempeña un papel importante sobre el producto de la concepción, es por esto que el peso preconcepcional, el IMC gestacional y la ganancia de peso son indicadores de amplio uso en la valoración nutricional de la gestante, que contribuyen al monitoreo del estado nutricional al inicio y durante la gestación para identificar el estado nutricional y de salud de la madre y de acuerdo con los resultados implementar un plan de alimentación acorde con los requerimientos, ofrecer educación nutricional ajustada a la cultura alimentaria y evaluar periódicamente los resultados de la intervención. (Restrepo \& Parra; 2009).

La preparación de los alimentos en el hogar debe proteger el valor nutricional, en particular el contenido de vitamina C. Como parte de la educación para la salud, se deben ofrecer algunas orientaciones prácticas, de forma sistemática, para así aumentar la biodisponibilidad de las vitaminas en los alimentos y del hierro en la dieta. (Mosquera; 2002). 


\section{La complejidad en la relación médico paciente: Una mirada actual}

Vol. 3, núm. 1., (2019)

Nakin Alberto Véliz Mero; Marco Vinicio Peñaherrera Alcívar; Martha Saida Quiroz Figueroa;

Hilda Monserrate Mendoza Sornoza; Jaime Eduardo Jaramillo Soto; Mónica Danny Tonguino

Rodríguez

Según Mosquera, (2002). La fuente alimentaria de hierro influye en gran medida sobre la eficiencia de su absorción, que oscila entre $<1 \%$ y $>20 \%$.

Contenido de hierro (mg) en $100 \mathrm{~g}$ de parte comestible.

$\begin{array}{llll}\text { Hígado de cerdo } & 29,1 & \text { Molleja de pollo } & 3,0 \\ \text { Riñón de res } & 13,0 & \text { Lengua de cerdo } & 3,0 \\ \text { Ajonjolí } & 10,0 & \text { Tamarindo } & 2,7 \\ \text { Hígado de pollo } & 8,5 & \text { Pato } & 2,7 \\ \text { Hígado de res } & 7,5 & \text { Pan corteza dura } & 2,5 \\ \text { Riñón de cerdo } & 6,6 & \text { Sesos de res } & 2,4 \\ \text { Chorizos } & 6,5 & \text { Jamón pierna } & 2,4 \\ \text { Perejil } & 6,2 & \text { Frijol (promedio) } & 2,4 \\ \text { Corazón de res } & 5,9 & \text { Frijol negro } & 2,2 \\ \text { Huevo de gallina } & & & \\ \text { (yema) de cerdo } & 5,5 & \text { Hamburguesa de carne } & 2,2 \\ \text { Corazón de } & 4,9 & \text { Lengua de res } & 2,2 \\ \text { Picadillo de res con } & & & \\ \text { soya } & 3,6 & \text { Maní } & 2,2 \\ \text { Hamburguesa } & & & \\ \text { soya con } & & \text { Lenteja } & 2,0 \\ \text { Carne de res magra } & 3,6 & \text { Huevo de gallina } & 2,0 \\ \text { Perro caliente } & 3,5 & \text { Mortadela Atabey } & 2,0\end{array}$

\section{Prevención de la anemia en el embarazo}

Las medidas llevadas a cabo para su prevención y manejo todavía son muy limitadas, particularmente en mujeres en edad reproductiva. Al respecto, la mayoría de los estudios se han focalizado en cuantificar su prevalencia y pocos son los que se enfocan en evaluar la eficacia y efectividad de las intervenciones para su prevención y control. (Casanueva,et al.; 2006).

En mujeres embarazadas, en donde el problema es mayor, algunos estudios han sugerido la suplementación con hierro y otros micronutrimentos; sin embargo, no se ha logrado la 


\section{La complejidad en la relación médico paciente: Una mirada actual}

Vol. 3, núm. 1., (2019)

Nakin Alberto Véliz Mero; Marco Vinicio Peñaherrera Alcívar; Martha Saida Quiroz Figueroa; Hilda Monserrate Mendoza Sornoza; Jaime Eduardo Jaramillo Soto; Mónica Danny Tonguino Rodríguez

efectividad deseada debido a lo tardío de la intervención durante el embarazo. (Scanlon et al.; 2000) y (Scholl; 2005).

El hierro es un nutriente esencial componente de muchas enzimas y proteínas necesarias para el metabolismo celular y el funcionamiento del organismo, dentro de las cuales se encuentra la hemoglobina, molécula encargada de transportar el oxígeno a todos los tejidos maternofetales. La gestante requiere altas cantidades de hierro para satisfacer las demandas por la síntesis acelerada de glóbulos rojos, la placenta, el crecimiento y el desarrollo fetal, además para reponer las pérdidas de este mineral durante el parto y el post parto. La necesidad de hierro aumenta casi linealmente hasta el término de la gestación y se calcula que la mujer requiere entre 800 y 900 mg durante el embarazo; en promedio, necesita absorber cerca de 5,6 mg por día durante el segundo y tercer trimestre, es decir 4,2 mg más que las mujeres no embarazadas. (Milman; 2006).

La deficiencia de hierro puede ser prevenida mediante el incremento del contenido y la biodisponibilidad del hierro en la dieta. En los países en desarrollo, donde la ingestión de carne es reducida, el ácido ascórbico aporta el refuerzo más importante para la absorción del hierro. El hierro de fuentes vegetales, en principio se absorbe peor, pero aunque sus reservas en los vegetarianos suelen ser inferiores, las tasas de anemias son similares a las de los no vegetarianos. (George; s/f).

Algunos organismos internacionales han propuesto cuatro estrategias para reducir o erradicar la anemia por deficiencia de hierro: fortificación de alimentos con hierro, 


\section{La complejidad en la relación médico paciente: Una mirada actual}

Vol. 3, núm. 1., (2019)

Nakin Alberto Véliz Mero; Marco Vinicio Peñaherrera Alcívar; Martha Saida Quiroz Figueroa;

Hilda Monserrate Mendoza Sornoza; Jaime Eduardo Jaramillo Soto; Mónica Danny Tonguino

Rodríguez

suplementación; promoción, comunicación y educación nutricional así como la vigilancia epidemiológica. (Scanlon; et al.; 2000).

Según Siega, et al., (2006), encontraron que el promedio de peso al nacer fue significativamente más alto, alrededor de 108 g, y la incidencia de parto pretérmino más baja, en el grupo que ingirió $30 \mathrm{mg}$ de hierro elemental por día, comparado con el grupo control sin suplemento del mineral. A pesar de la amplia evidencia de estudios observacionales que asocian la anemia materna con el tamaño del recién nacido, la duración de la gestación y la mortalidad neonatal o perinatal, se requieren más estudios para definir los puntos de corte utilizados que permitan determinar una hemoglobina normal, baja o alta, precisar las semanas de gestación de la madre al momento de determinar este indicador y asociarlo con resultados de la gestación como el peso al nacer, debido a que los cambios hemodinámicos durante este período tienen una importante influencia sobre la concentración de esta proteína.

Esta cantidad es difícil de satisfacer a partir de fuentes naturales de alimentos, todavía más en los países pobres donde la base de la alimentación son los cereales y por tanto las fuentes de hierro son de baja biodisponibilidad, principal razón por la cual organismos internacionales como la OMS recomiendan un suplemento con dosis moderadas de este mineral para satisfacer las recomendaciones diarias, prevenir la anemia ferropénica y evitar las repercusiones negativas para la salud de la madre y el feto. (Parra; 2007), (Ren et al; 2007).

Al respecto, México ha implementado programas de suplementación con hierro y ácido fólico a mujeres embarazadas. Además, cuenta con programas sociales que distribuyen alimentos fortificados con hierro y otros micronutrimentos a la población en pobreza; como el 


\section{La complejidad en la relación médico paciente: Una mirada actual}

Vol. 3, núm. 1., (2019)

Nakin Alberto Véliz Mero; Marco Vinicio Peñaherrera Alcívar; Martha Saida Quiroz Figueroa; Hilda Monserrate Mendoza Sornoza; Jaime Eduardo Jaramillo Soto; Mónica Danny Tonguino Rodríguez

complemento fortificado del programa Oportunidades y la leche fortificada Liconsa del

Programa de Abasto Social de Leche; cuyos efectos han mostrado una disminución importante de la prevalencia de anemia en los niños. Es importante continuar con las estrategias de suplementación y de fortificación de alimentos que den cobertura universal a las mujeres en edad reproductiva; así como evaluar la efectividad de dichos programas en este sector de la población. Paralelamente, resulta necesario identificar y actualizar la contribución de las causas (nutricionales y no nutricionales) de la anemia en esta población. (Shamah-Levy et al.; 2013).

El Ministerio de Salud de Bolivia, con ayuda del Programa Mundial de Alimentos (PMA), tiene como estrategia la “Asistencia a la Ejecución del Programa Nacional de Prevención de Control de las Anemias Nutricionales" a través de la dotación, a las mujeres embarazadas y puérperas, de 90 tab. de sulfato ferroso compuesto, en una concentración de 60 mg. de hierro en la forma de sulfato ferroso y $5 \mathrm{mg}$. ácido fólico. Dicha estrategia ha sido evaluada el 2005, donde se han entregado 90 tab. de sulfato ferroso a 152.476 embarazadas de las 317.501 (48\%). (Ministerio de Salud y Deportes; 2007).

El problema más común en el tratamiento de la deficiencia de hierro son los efectos secundarios, los más frecuentes son los gastrointestinales. El riesgo de dichos efectos secundarios es directamente proporcional a la dosis del metal, y los síntomas suelen atribuirse a la administración de proporciones mayores de las necesarias de compuestos de hierro con >120 mg/día de hierro elemental. El equivalente a una dosis total de $60 \mathrm{mg}$ de hierro elemental al día es suficiente para un adulto si se administra entre las comidas, antes de desayunar o al acostarse. (Yip, R.; 2003) 


\section{La complejidad en la relación médico paciente: Una mirada actual}

Vol. 3, núm. 1., (2019)

Nakin Alberto Véliz Mero; Marco Vinicio Peñaherrera Alcívar; Martha Saida Quiroz Figueroa;

Hilda Monserrate Mendoza Sornoza; Jaime Eduardo Jaramillo Soto; Mónica Danny Tonguino

Rodríguez

Por fortuna, cuanto menor sea la dosis y más grave la anemia, mayor será el porcentaje de

hierro absorbido. Al cabo de 1 mes, la respuesta al tratamiento debe ser evidente, con corrección parcial del déficit de hemoglobina y ascenso de su valor por encima de $100 \mathrm{~g} / \mathrm{L}$. Aunque la respuesta haya sido buena, deberá mantenerse la administración de hierro durante otros 2 ó 3 meses. Si después de 1 mes de tratamiento la anemia no se hubiera corregido, debe indicarse un estudio de laboratorio más amplio (puede ser con ferritina sérica) para confirmar la presencia de deficiencia de hierro o determinar otras causas de anemia. La absorción de hierro interactúa con la del $\mathrm{Zn}, \mathrm{Cu}, \mathrm{Co}, \mathrm{Ca}$ y otros; una ingesta excesiva de dicho mineral, como suplemento farmacéutico, puede ocasionar una deficiencia de zinc y ser antagonista del cobre, por lo que debe evitarse su consumo exagerado en forma de preparación medicamentosa. (Yip, R.; 2003).

La deficiencia de hierro se puede prevenir mediante modificaciones de la dieta, fortificación de los alimentos y suplementación con hierro nutricional. Ninguna de estas estrategias es excluyente. La forma ideal de prevenir la carencia de hierro es mediante una dieta adecuada, lo que no siempre es posible de lograr por limitaciones económicas o hábitos muy arraigados. (OPS; 2004).

Es necesario promover el consumo de alimentos biodisponibles en hierro (como las carnes) mediante la educación nutricional y difundir información sobre las consecuencias en la salud que conlleva la anemia y la deficiencia de hierro. Adicionalmente promover y apoyar la lactancia materna exclusiva para mantener las reservas corporales de hierro después del parto, manteniendo el binomio lactancia-amenorrea en aquellas mujeres no anémicas. En las anémicas, se sugiere suplementar con hierro. (Shamah-Levy et al.; 2013). 


\section{La complejidad en la relación médico paciente: Una mirada actual}

Vol. 3, núm. 1., (2019)

Nakin Alberto Véliz Mero; Marco Vinicio Peñaherrera Alcívar; Martha Saida Quiroz Figueroa; Hilda Monserrate Mendoza Sornoza; Jaime Eduardo Jaramillo Soto; Mónica Danny Tonguino Rodríguez

Según Gay, (1998), Las dietas se clasifican en 3 categorías, según su biodisponibilidad: baja, intermedia o alta; con una absorción media de hierro, aproximadamente, de 5, 10 y $15 \%$, respectivamente:

- Biodisponibilidad baja: una dieta monótona, compuesta por cereales, raíces, tubérculos y cantidades insignificantes de carnes, pescado o alimentos ricos en ácido ascórbico.

- Biodisponibilidad intermedia: compuesta principalmente por cereales, raíces, tubérculos y cantidades moderadas de ácidos ascórbico, carnes o pescados. Una dieta de biodisponibilidad baja puede convertirse en intermedia, si se aumenta la ingesta de alimentos que mejoren la absorción del hierro y, por el contrario, una de intermedia puede convertirse en baja, si se consumen, regularmente, en una misma comida del día, cantidades mayores de inhibidores de la absorción del hierro, como el té o café.

- Biodisponibilidad alta: dieta diversificada que contenga cantidades amplias de carnes, aves, pescado y alimentos ricos en ácido ascórbico.

La primera línea de tratamiento en la mayoría de las pacientes es la reposición con hierro oral debido a su eficacia, seguridad y bajo costo. En estos casos, según American College of Obstetricians and Gynecologists (ACOG), se debe proveer un aporte diario de hierro elemental de 160-200 mg/día. De esta manera casi siempre se logra una rápida respuesta, con un incremento promedio de la $\mathrm{Hb}$ de $1 \mathrm{~g} / \mathrm{dL}$ después de 14 días de tratamiento efectivo. Esta recomendación también aplica para los casos de deficiencia de hierro sin anemia (niveles de $\mathrm{Hb}$ normales), frecuentes al inicio del embarazo. Bhavi \& Jaju, (2017). 


\section{La complejidad en la relación médico paciente: Una mirada actual}

Vol. 3, núm. 1., (2019)

Nakin Alberto Véliz Mero; Marco Vinicio Peñaherrera Alcívar; Martha Saida Quiroz Figueroa;

Hilda Monserrate Mendoza Sornoza; Jaime Eduardo Jaramillo Soto; Mónica Danny Tonguino

Rodríguez

En la tabla 1 se especifican las concentraciones de hierro elemental de las diferentes presentaciones de hierro oral. (ACOG; 2008).

Tabla 1. Presentaciones de hierro oral

\begin{tabular}{|c|c|c|}
\hline Presentación & Tableta (mg) & $\begin{array}{c}\text { Hierro elemental } \\
\text { (mg) }\end{array}$ \\
\hline Sulfato ferroso & 325 & 65 \\
\hline Gluconato ferroso & 300 & 34 \\
\hline Fumarato ferroso & 325 & 106 \\
\hline
\end{tabular}

Fuente: ACOG, (2008).

De igual forma, varios estudios han mostrado que el hierro parenteral, cuando está indicado, es superior al hierro oral al comparar la velocidad y la magnitud del incremento de las cifras de hemoglobina. De igual forma, evitan los problemas de tolerancia asociados a las formas orales; sin embargo, los severos efectos adversos sistémicos asociados con las presentaciones parenterales han limitado su uso, lo que se asocia a reacciones anafilácticas severas en $1 \%$ de las pacientes. (ACOG; 2008).

En la actualidad, se recomienda el uso de hierro parenteral en las siguientes circunstancias:

No respuesta a la terapia con hierro oral (niveles de $\mathrm{Hb}<10 \mathrm{~g} / \mathrm{dL}$ después de 14 días de tratamiento efectivo).

- Intolerancia a formulaciones orales o poca adherencia.

- Anemia severa $(\mathrm{Hb}<9 \mathrm{~g} / \mathrm{dL})$. 


\section{La complejidad en la relación médico paciente: Una mirada actual}

Vol. 3, núm. 1., (2019)

Nakin Alberto Véliz Mero; Marco Vinicio Peñaherrera Alcívar; Martha Saida Quiroz Figueroa; Hilda Monserrate Mendoza Sornoza; Jaime Eduardo Jaramillo Soto; Mónica Danny Tonguino Rodríguez

- Necesidad de tratamiento eficaz rápido (edad gestacional avanzada, placenta previa, testigos de Jehová, etc.)

Pacientes con síndrome de malabsorción

Por lo tanto, en la mayoría de circunstancias clínicas, el hierro oral es la intervención terapéutica más adecuada y en pocas circunstancias es necesario el uso de hierro parenteral. En la Tabla 2, se enuncian algunas de las presentaciones disponibles de hierro parenteral. (ACOG; 2008).

Tabla 2. Presentaciones parenterales de hierro

\begin{tabular}{|c|c|c|}
\hline Presentación & Vía & Hierro elemental (mg/ml) \\
\hline Hierro dextrán & IV o IM & 50 \\
\hline Gluconato férrico & IV & 12,5 \\
\hline Fumarato ferroso & IV & 20 \\
\hline
\end{tabular}

Fuente: ACOG, (2008). IV (Intravenoso), IM (Intramuscular)

El Ministerio de Sanidad, Política Social e Igualdad en España, recomienda la ingesta de un suplemento de 0,4 mg de ácido fólico al día a toda mujer que esté planeando un embarazo. Aquellas con antecedentes de DTN la dosis serán de 4 mg/día. Se deberá empezar al menos un mes antes de la concepción, y mantenerse como mínimo hasta el final del primer trimestre. La tendencia actual es mantenerlo durante todo el embarazo, sobre todo en casos de gemelaridad, enfermedades crónicas, vómitos de repetición o malabsorción de cualquier origen. La toma debe 


\section{La complejidad en la relación médico paciente: Una mirada actual}

Vol. 3, núm. 1., (2019)

Nakin Alberto Véliz Mero; Marco Vinicio Peñaherrera Alcívar; Martha Saida Quiroz Figueroa;

Hilda Monserrate Mendoza Sornoza; Jaime Eduardo Jaramillo Soto; Mónica Danny Tonguino

Rodríguez

ser diaria e ininterrumpida, porque no se almacena en el organismo. Su consumo irregular disminuye o anula el efecto protector. (López et al.; 2010).

En cuanto a los posibles riesgos de niveles elevados de ácido fólico estaría la posibilidad de que se enmascare una anemia perniciosa, poco probable con las dosis habituales de suplementación. El nivel de seguridad se establece en $1 \mathrm{mg}$ /día. Además se aconseja tomarlo junto con vitamina $\mathrm{B}_{12}$. Independientemente de esto, se aconseja a todas las embarazadas la ingesta de alimentos que aporten ácido fólico, especialmente vegetales de hoja verde, hígado, frutas, cereales, legumbres, levaduras y frutos secos. (López et al.; 2010).

\section{Conclusiones.}

En general a fin de reducir o erradicar la anemia por deficiencia de hierro se recomienda a las a las mujeres en edad reproductiva, prevenir mediante modificaciones de la dieta, fortificación de los alimentos y suplementación con hierro nutricional. Ninguna de estas estrategias es excluyente. La forma ideal de prevenir la carencia de hierro es mediante una dieta adecuada, lo que no siempre es posible de lograr por limitaciones económicas o hábitos muy arraigados. Es necesario promover el consumo de alimentos biodisponibles en hierro (como las carnes) mediante la educación nutricional y difundir información sobre las consecuencias en la salud que conlleva la anemia y la deficiencia de hierro. Adicionalmente promover y apoyar la lactancia materna exclusiva para mantener las reservas corporales de hierro después del parto, se recomiendan los suplementos de dosis bajas de hierro oral durante la segunda mitad del embarazo en las mujeres sin riesgo de ferropenia. En pacientes con anemia previa se debe iniciar al principio de la gestación. Es importante considerar en el tratamiento de la deficiencia de 


\section{La complejidad en la relación médico paciente: Una mirada actual}

Vol. 3, núm. 1., (2019)

Nakin Alberto Véliz Mero; Marco Vinicio Peñaherrera Alcívar; Martha Saida Quiroz Figueroa; Hilda Monserrate Mendoza Sornoza; Jaime Eduardo Jaramillo Soto; Mónica Danny Tonguino Rodríguez

hierro, los efectos secundarios, siendo los más frecuentes los gastrointestinales. El riesgo de dichos efectos secundarios es directamente proporcional a la dosis del metal, y los síntomas suelen atribuirse a la administración de proporciones mayores de las necesarias de compuestos de hierro.

\section{Bibliografía.}

Abrams, B., Altman, S., Pickett, K., (2000), Pregnancy weight gain: still controversial, Am J Clin Nutr. 71 (suppl) 1233S.

ACOG, (2008), Anemia in pregnancy, American College of Obstetricians and Gynecologists (ACOG), Practice Bulletin No. 95: Obstet Gynecol. 112 (1): 201-7. doi: 10.1097/AOG.0b013e3181809c0d

Anivarro, P., (2007), Prevalencia de anemia en pacientes asegurados y beneficiarios de los Servicios de Salud-Policlínico 9 de abril, Caja Nacional de Salud, La Paz-Bolivia, Rev. Paceña Med. Fam. 4(5): 3-14.

Bhavi, S. \& Jaju, P., (2017), Intravenous iron sucrose v/s oral ferrous fumarate for treatment of anemia in pregnancy, A randomized controlled trial, BMC Pregnancy Childbirth, 17(1): 137, doi: 10.1186/s12884-017-1313-9.

Casanueva, E., De Regil, L., Flores, M., (2006), Anemia por deficiencia de hierro en mujeres mexicanas en edad reproductiva, Historia de un problema no resuelto, Salud Publica México, 48: 166-175.

Espitia, F. \& Orozco, L., (2013), Anemia en el embarazo, un problema de salud que puede prevenirse, Méd. UIS. 2013, 26(3): 45-50.

Gay, J., (1998), Prevención y control de la carencia de hierro en la embarazada. Revista Cubana Aliment Nutr 1998; 18(2): 15-8.

George Ecismen, R., (s/f), La buena nutrición: una mirada a lo básico del vegetarianismo, $<$ http://www.ivu.org/spanish/trans/naus - nutri.html>

Hernández P., (2006), Frecuencia de anemia en recién nacidos a término en el hospital regional universitario tesis que para obtener el diploma de especialidad en pediatría, México 2006 p. 30-4.

Kilpatrick, S., Papile, L., Macones, G., (2017), Pautas para el cuidado perinatal, American Academy of Pediatrics, American College of Obstetricians and Gynecologists editores. 


\section{La complejidad en la relación médico paciente: Una mirada actual}

Vol. 3, núm. 1., (2019)

Nakin Alberto Véliz Mero; Marco Vinicio Peñaherrera Alcívar; Martha Saida Quiroz Figueroa;

Hilda Monserrate Mendoza Sornoza; Jaime Eduardo Jaramillo Soto; Mónica Danny Tonguino Rodríguez

8va. Edición, Washington, DC: American Academy of Pediatrics ; The American College of Obstetricians and Gynecologists.

López N. \& Alvarez R., (2010), Anemia muy precoz del prematuro con peso $\leq 1500$ g: prevalencia y factores asociados, Rev Infectología y Enfermedades Tropicales, 82(2).

López Rodríguez, M., Sánchez Méndez, J., Sánchez Martínez, M., Calderay Domínguez, M., (2010), Suplementos en embarazadas: controversias, evidencias y recomendaciones, Inf. Ter. del Sistema Nacional de Salud. Volumen 34, No 4, pp. 117-128.

Manjarrés, L., Parra, B., Díaz, A., Restrepo, S., Mancilla, L., (2012), Ingesta de hierro y folatos durante el embarazo y su relación con indicadores bioquímicos maternos. Iatreia. 25(3): 194-202.

Martínez, L., Jaramillo, L., Villegas, J., Álvarez, L., Ruiz, C., (2018), La anemia fisiológica frente a la patológica en el embarazo, Editorial Ciencias Médicas, Vol. 44, Núm. 2, ISSN 1561-3062, http://www.revginecobstetricia.sld.cu/index.php/gin/article/view/356/287

Mei, Z., Cogswell, M., Parvanta, I., Lynch, S., Beard, J., Stoltzfus, R., et al. (2005), Hemoglobin and ferritin are currently the most efficient indicators of population response to iron interventions: an analysis of nine randomized controlled trials, J Nutr. 135: 1974-80.

Milman, N., (2006), Iron and pregnancy--a delicate balance, Ann Hematol, 559-65.

Ministerio de Salud y Deportes, (2007), Programa desnutrición cero encuesta nacional de nutrición según niveles de vulnerabilidad a la inseguridad alimentaria, Bolivia.

Mosquera Aguelles, M., (2002), Guías alimentarias para la población cubana mayor de 2 años.: Casa Editora Abril, 2002, La Habana, 8-10.

OMS, (2003), Salud reproductiva, Organización Mundial de la Salud (OMS), OPS, 2003, Washington, DC.

OPS, (2004), Boletín Epidemiológico, Organización Panamericana de la Salud, 23 (3).

Parra, B., (2007), Evaluación bioquímica del estado nutricional del hierro y el folato en un grupo de mujeres gestantes participantes del programa MANA para la vida. En: Restrepo S, ed. Alimentación y nutrición de la mujer gestante: diagnóstico y lineamientos para la acción, Medellín: Gobernación de Antioquia. p. 217-43.

Peralta, M. \& Carbajal, P., (2008), Adherencia a tratamiento, Rev. Centro Dermatológico pascua 2008; 17: $84-88$.

Ramírez, J.V., (2009), Patología materna y embarazo, OBSTETRICIA- TEMA 26/1 https://www.uv.es/passats/obstetricia 


\section{La complejidad en la relación médico paciente: Una mirada actual}

Vol. 3, núm. 1., (2019)

Nakin Alberto Véliz Mero; Marco Vinicio Peñaherrera Alcívar; Martha Saida Quiroz Figueroa; Hilda Monserrate Mendoza Sornoza; Jaime Eduardo Jaramillo Soto; Mónica Danny Tonguino Rodríguez

Ren, A, Wang, J., Ye, R., Li, S., Liu, J., Li, Z., (2007), Low first-trimester hemoglobin and low birth weight, preterm birth and small for gestational age newborns, Int J Gynaecol Obstet. 98: 124-8.

Restrepo, S., Parra, B., (2009), Implicaciones del estado nutricional materno en el peso al nacer del neonato, Perspect Nutr Humana, 11: 179-86.

Roca Goderich, R., (2002), Temas de Medicina Interna, 4 edición, Editorial Ciencias Médicas, La Habana, t3: 321-24.

Siega, A., Hartzema, A., Turnbull, C., Thorp, J., McDonald, T., Cogswell, M., (2006), The effects of prophylactic iron given in prenatal supplements on iron status and birth outcomes: a randomized controlled trial, Am J Obstet Gynecol, 194: 51.

Scanlon, K., Yip, R., Schieve, L., Cogswell, M., (2000), High and low hemoglobin levels during pregnancy: differential risks for preterm birth and small for gestational age, Obstet Gynecol, 96: 741-748.

Scholl, T., (2005), Iron status during pregnancy: setting the stage for mother and infant, Am J Clin Nutr. 81: 218S-1222S.

Shamah-Levy, T., Villalpando, S., Mundo-Rosas, V., De la Cruz-Góngora, V., Mejía-Rodríguez, F., Gómez-Humarán, I., (2013), Prevalencia de anemia en mujeres mexicanas en edad reproductiva, 1999-2012, salud pública de méxico / vol. 55, suplemento 2 de 2013.

Silva, G., Galeano, E., Correa, J., (2005), Adherencia al Tratamiento, Acta médica de Colombia, 30: $268-273$.

Tojo, R., (2001), Tratado de Nutricion Nediatrica, Nutrición de la madre gestante y del lactante, Doyma, Barcelona, p.337 - 338 .

Urdampilleta, A., Martínez, J., González, P., (2010), Intervención dietético Nutricional en la prevención de la deficiencia de hierro Nutr. Clin. Diet. Hosp. 30(3), pp.27-41 <http://www.nutrición.org/para-saber-mas/dietoterapia- anemia.htm>

Yip, R., (2003), Hierro. En: Conocimientos actuales sobre nutrición, Organización Panamericana de la Salud, 8 edición, Washington, DC: 340-56.

WHO, (2011), Haemoglobin concentrations for the diagnosis of anaemia and assessment of severity, World Heath Organization, Geneva: Disponible en: http://www.who.int/vmnis/indicators/haemoglobin/en/

WHO, (2001), Iron deficiency anaemia: assessment, prevention and control, World Heath Organization, WHO, página 114, Disponible en: http://www.who.int/nutrition/publications/micronutrients/anaemia_iron_deficiency/WHO _NHD_01.3/en 